\section{Ethics and Aging - The Right to Live, the Right to Die}

\author{
Edited by James E Thornton and Earl \\ R Winkler, 256 pages, Vancouver, \\ $£ 13.55$ pbk, University of British \\ Columbia Press, 1988
}

'For a variety of historical and cultural reasons, we presently confront a vast array of practical and moral problems and decisions concerning the status, role and welfare of the elderly in society'. Thus this book describes and contributes to the ethical clarification needed to respond to this challenge in the present climate of 'general concern about individual rights, and claims - to autonomy, health care, equality and so forth'.

The editors, from educational gerontology and philosophy at the University of British Columbia, were instrumental in the creation of a symposium on ethics and aging in 1982. Some contributions derive from the symposium, others were invited subsequently to broaden the book's scope. The urgency of tackling these problems derives not solely from changes in population structure but also from evolving family structure, the mobility of working populations, the impact of retirement on individuals and societies and medical technological developments. This breadth is reflected in the authorship which spans gerontology, philosophy, law, theology, sociology, psychology, medicine, nursing and economics. What emerges is not an untidy hybrid but an invigorating debate, clarification coming as much from conflict as from consensus.

The early chapters provide a factual and conceptual background for the later discussion of specific issues. The shortfall of current health care and social programmes is explored, in terms of quantity and also with regard to questions of professional dominance, against the background of misconceptions about and marginalisation of elderly people themselves. The characterisation of aging as decline rather than growth is contested from recent evidence and fresh interpretation of psychological research.

A detailed analysis of the economic implications of the demographic changes provides cautious optimism that the task involves a planned reallocation of resources rather than a substantial increase. With regard to the very topical issue of making priorities in provision of health care, $\mathrm{E} \mathrm{H} \mathrm{W} \mathrm{Kluge}$ makes a convincing rebuttal of current approaches such as cost-effectiveness and cost-benefit analysis, on the grounds that discrimination is based upon the abstract category of age rather than ethically relevant differences between individuals.

Many contributors tackle the 'withdrawal of treatment'. One, a theologian, was the only octogenarian in the assembled cast. The contrasting outcome of the Canadian Law Reform Commission and the (Canadian) President's Commission on the subject are critically discussed from the point of view of ethical content and consistency.

A most interesting and useful chapter explores the question of consent for research with 'incompetent elderly', for example those suffering from Alzheimer's disease, and goes some way towards establishing a practical approach for researchers and ethics committees. A major problern in the resolution of many of the ethical problems posed is that of the lack of involvement or control held by elderly people themselves, but a contribution on gerontological research with elderly people as research collaborators shows the way forward.

This book is Canadian in conception and authorship but the problems tackled and the methods used have no nationality.

I thoroughly enjoyed reading it, but more important, the book has changed my understanding of what I do in daily clincal practice.

I thoroughly recommend it not only to clinicians working with elderly people, but also to administrators, planners and politicians.

FINBARR MARTIN Consultant Physician Department of Medicine for the Elderly St Thomas's Hospital London SE 1

\section{Strong Medicine - Health Politics for the Twenty-first Century}

Steve Iliffe, 157 pages, London, £5.95, Lawrence and Wishart, 1988

It seems slightly unfair to read and review Steve Iliffe's political essays in the days following the publication of the NHS Review, Working for Patients. Overnight, the whole debate on the future of the NHS and the political and social implications for the next decade have been shifted from one level of uncertainty to another, leaving $\mathrm{Dr}$ Iliffe's book looking rather dated in detail if not in spirit.

In particular, the first section of the book in which Dr Iliffe attempts to predict the political consequences of the crisis in the NHS now seems irrelevant to the fundamental arguments of the ethical development and defence of health provision into the next century. What is more relevant is the review of the foundation of the National Health Service and the egalitarian principle of 